\title{
Evaluation of temporomandibular disorder and oral parafunction prevalence in dental assistant students: A cross-sectional descriptive study
}

\author{
Taner Öztürk ${ }^{1}$ and Ravza Eraslan ${ }^{1}$ \\ ${ }^{1}$ Erciyes University Faculty of Dentistry
}

July 5, 2021

\begin{abstract}
Objective: This cross-sectional descriptive study aimed to determine the prevalence of temporomandibular disorder (TMD) and oral parafunctions in dental assistant students and examine the effects of sex and years of education. Materials and Methods: One-hundred five dental assistant students were included in the study. The Fonseca Anamnestic Index (FAI) was used to evaluate the presence of TMD in individuals, and the Oral Habit Checklist (OBC) was used to evaluate oral parafunctions. Results: Sixty-eight individuals $(64.8 \%)$ had TMD at some level. It was determined that 46 of the females, who constituted the majority of the participants, had mild-TMD, which was significantly different from the males ( $<0.001)$. According to the OBC results, oral parafunctions were associated with TMD, with low-moderate significant correlations $(\mathrm{p}<0.05)$. Conclusion: Early diagnosis of temporomandibular joint problems with various questionaries in the university, especially in the young population, is vital in terms of disease prognosis and will prevent future complications.
\end{abstract}

Evaluation of temporomandibular disorder and oral parafunction prevalence in dental assistant students: A cross-sectional descriptive study

Short Title: TMD 8 Oral Parafunctions in Dental Assistant Students

Abstract

Objective: This cross-sectional descriptive study aimed to determine the prevalence of temporomandibular disorder (TMD) and oral parafunctions in dental assistant students and examine the effects of sex and years of education.

Materials and Methods: One-hundred five dental assistant students were included in the study. The Fonseca Anamnestic Index (FAI) was used to evaluate the presence of TMD in individuals, and the Oral Habit Checklist (OBC) was used to evaluate oral parafunctions.

Results : Sixty-eight individuals (64.8\%) had TMD at some level. It was determined that 46 of the females, who constituted the majority of the participants, had mild-TMD, which was significantly different from the males $(p<0.001)$. According to the OBC results, oral parafunctions were associated with TMD, with low-moderate significant correlations $(p<0.05)$.

Conclusion: Early diagnosis of temporomandibular joint problems with various questionaries in the university, especially in the young population, is vital in terms of disease prognosis and will prevent future complications.

Keywords : dental assistant, oral parafunctions, temporomandibular disorder 


\section{What's known}

- The prevalence of TMD and oral parafunction has been described in many different areas of healthcare.

- In dentistry, the awareness and number of dental assistants who assist clinicians in clinics and many institutions is increasing.

- Dental assistants have a significant role in the execution of dental services.

- Oral health findings in dental assistant students and dentistry students should be evaluated and revealed.

\section{What's new}

This study revealed a relationship between the prevalence of TMD and oral parafunction in individuals who are trained for dental assistant, and it is thought that there should be an awareness of TMD and oral parafunctions in this student population.

Although there are varying degrees of correlation between TMD and oral parafunctional, the relationship between FAI and OBC scores has been fully demonstrated.

\section{Introduction}

The temporomandibular joint (TMJ) is one of the most complex joints between the mandible and the temporal bones of the skull and is responsible for the chewing, opening, and closing movements of the mouth, as well as the protrusion, retrusion, and lateral deviation of the mandible. ${ }^{1,2}$ Problems in this joint can be defined as a multifactorial joint dysfunction characterized by pain in the oro-facial region, fatigue in the craniofacial and cervicofacial muscles, spasm, limitation of movement in the mandible due to the influence of the masticatory muscles, and a "click" sound during joint movements. ${ }^{3}$ Temporomandibular disorder (TMD) etiology is multifactorial, such as emotional and psychological problems, occlusal incompatibilities, prolonged dental treatments and spasm in the chewing muscles due to oral habits, a trauma in the craniofacial region, false chewing patterns due to early tooth loss, aging, clenching, and grinding, malfunction in anatomical structures adjacent to TMJ area. ${ }^{4}$

Symptoms such as pain, which arise due to TMD disorders, can cause irreversible problems in the future by making the treatment process difficult due to not diagnosed in the early period, affecting the daily activities and psychosocial quality of life of the individuals. ${ }^{5,6}$ Early diagnosis of TMD, which has a multifactorial mechanism of formation, is of great importance to prevent the emergence of problems that are very difficult to compensate, and the treatment process is quite complex. ${ }^{7}$ It has been shown that the severity of TMD symptoms can vary according to age, sex, and educational background. ${ }^{8-10}$ Some studies on the prevalence of TMD report the prevalence in the population to be $50-70 \%$, differing according to racial changes, the size of the population used, the diagnostic criteria, and the method of examination. ${ }^{11-13}$ Most of the epidemiological studies conducted on determining the prevalence of TMD have been conducted on university students studying any field related to health services. ${ }^{1,3,5,14-17}$ It has been reported that the prevalence of TMD shows a wide range between $47-81 \%$ in studies conducted on students from the Turkish population, with studies conducted in some of the populations. ${ }^{15-19}$ TMD problems have been reported to be on the rise in the adolescent and adult population. ${ }^{9,20,21}$ It is known that oral parafunctional behaviors, such as clenching and/or grinding; prolonged nail, lip, cheek, pencil sucking and/or biting; and frequent gum chewing, play an important role in the etiology of TMD. ${ }^{22}$ Therefore, in various studies in the literature, the prevalence and relationship between TMD and oral parafunction were examined. ${ }^{5,7,16,19}$ However, it is still needed to determine which oral behaviors caused TMD and further work to examine the relationship between them. ${ }^{21}$

TMD problems, which are often encountered in a wide range of ages and different populations, can sometimes be available as an asymptomatic character without the painful finding of patients who consult the physician. For this reason, the early diagnosis of TMD does not revert. Although there are very different evaluation methods in the literature, ${ }^{22-24}$ various surveys are used to determine factors, such as rapid, practical, cost, and reliability, that can be easily understood by individuals and contribute to the high TMD and contribute 
to the formation of factors such as parafunctional habits, motion limitation, climatic sound, and emotional stress. ${ }^{16,25}$ The Fonseca Amnestic Index (FAI) is commonly used for TMD-related evaluation. ${ }^{12,13}$ It is often used to determine parafunctional oral habits. The Oral Behaviors Checklist (OBC) is also frequently used. ${ }^{26}$

There are no studies on TMD prevalence and awareness, and oral parafunction among dental assistant program students. This study aimed to evaluate the presence of TMJ problems in dental assistant program students and determine the relationship between these problems and oral behaviors.

\section{Material and Methods}

The Approval (approval code: 2021/88) was obtained from the XXX University Clinical Research Ethics Committee for this descriptive and cross-sectional study, which evaluated TMD and oral habits of dental assistant student volunteers who selected from XXX University Vocational Health College between January 15, 2021, and February 15, 2021. In the period when the study was implemented, to prevent a possible virus transmission due to the coronavirus disease 2019 (COVID-19) pandemic, the questionnaire questions were delivered to the volunteers, using the Google Forms internet question application system and following the social distance rules, face-to-face but online marking (27). During the study, 25 out of 130 students in the dental assistant education program were excluded from the study because 15 of 25 students did not answer the questions, and the remaining ten students could not be reached. Responses were received from 105 participants at the end (Figure 1). A total of 105 volunteers (mean age: $20.72 \pm 3.51$ years), 30 males (mean age: $22.60 \pm 5.94$ years) and 75 females (mean age: $19.97 \pm 1.21$ years), were included in the study.

Participants were informed about the objectives, risks, and benefits of the study before the administration of the questionnaires, and verbal and online informed consent was obtained from each participant, marking their admission to the study. Volunteers' data were obtained using the FAI ${ }^{13}$ to determine the status and severity of TMD and the $\mathrm{OBC}^{26}$ to examine the characteristics and severity of oral habits.

Sample size calculation

Considering the correlation of 0.505 between FAI and OBC determined in the study conducted by Karabicak and $\mathrm{Kanik}^{19}$, it was determined that at least 45 cases should be investigated with $95 \%$ confidence $(1-\alpha)$, $95.4 \%$ test power $(1-\beta)$, and $\rho=0.505$ effect size.

\section{Outcome measures}

The Turkish version of the 10-question questionnaire of Fonseca, which included factors such as chewing, parafunctional habits, movement limitations, joint noise, and dizziness, was applied to all volunteers and was presented as a low-cost and easy-to-apply alternative to TMD findings. ${ }^{12,16,19}$ For each of the ten questions prepared as a three-point Likert scale, "yes, no, and sometimes" answer options were given. Students were asked to mark only one option without any time limitation, with 10 points for each "yes" answer, 5 points for "sometimes," and 0 points for "no" answers. The FAI was used to determine the severity of the TMJ. After collecting the score values of the answers given to all questions, individuals were divided into four groups according to the signs and symptoms related to TMD. The classification of the groups and their distribution according to the scores were as follows: 0-15 points, no symptoms; 20-45 points, mild TMD; 50-65 points, moderate TMD; and 75-100 points, severe TMD group. ${ }^{12}$

To examine the oral parafunctional habits of the participants, the Turkish version of the OBC questionnaire, which has a comprehensive 21-question scale with a 5 -item Likert-type scale for each item, has good validity and reliability for wakeful behavior. ${ }^{5,16,26,28}$ Participants wrote each item according to the frequency of complaints " $4=$ always; $3=$ frequently; $2=$ sometimes; $1=$ rarely or $0=$ never" (19). The total score of $\mathrm{OBC}$ was used for analysis. A person's overall score ranged from 0 to 84 .

In addition, the three following questions prepared by the researchers were asked to determine the awareness of the volunteers included in the study about the possible TMD status or the treatments they received: "Do you think you have a problem with the jaw/jaw joint?;" "Have you applied to the dentist for jaw/jaw joint pain in the last 1 year?;" and "Any treatment for jaw/jaw joint pain in the last 1 year (mouthpiece, exercise, 
medication..., etc.) have you seen?." They were asked to answer the three questions posed as "yes" and "no". ${ }^{14,18}$

\section{Statistical analysis}

The data obtained were stored and processed using Microsoft Excel (Microsoft 365, Microsoft Office, Microsoft, NY, USA). Statistical analysis of the data was performed using SPSS (Statistical Package of Social Sciences, Ver. 24.0 IBM, Armonk, NY, USA). The chi-square test was used to examine the relationship between sex and class in the distribution of the TMD score, and the Mann-Whitney U test was used to compare OBC scores by sex and class. The Kendall tau-b correlation test was used to evaluate the relationship between $\mathrm{OBC}$ and the Fonseca index. To define the strength of the correlation between variables, those between $0-0.10$ are negligible, those between $0.10-0.39$ are weak, those between $0.40-0.69$ are medium, those between $0.70-0.89$ are strong, and those between $0.90-1.00$ are classified as having very strong correlations. ${ }^{29}$ The Linear regression analysis was carried out using the Enter method to determine the mathematical relationship between FAI and OBC scores, which are significantly correlated. A $p$-value of less than 0.05 was considered statically significant.

\section{Results}

One hundred five students, 75 females $(19.97 \pm 1.21$ years $)$ and 30 males $(22.60 \pm 5.94$ years $)$ from XXX University Health Vocational College participated in this study $(\mathrm{n}=105)$. The mean age of the participants was $20.72 \pm 3.51$ years. Sixty-eight participants $(64.8 \%$ ) had TMJ problems at some level, among them $74.3 \%$ $(\mathrm{n}=52)$ were female and $36.7 \%(\mathrm{n}=11)$ were males. The prevalence of TMD was statistically significantly higher in females than in males (Table 1$)(p<0.05)$. No significant difference was found between the TMD prevalence of the first-and second-grade students based on the years of education of the participants (Table $2, p=0.614)$.

It was observed that the score values of females were significantly higher than those of males (Table $3, p=$ 0.025). There was no statistically significant difference in the OBC score severity class parameters (Table 3 ). Considering the sex and class parameters, a statistically significant positive correlation between the OBC scores severity and TMD scores for both parameters $(\mathrm{r}=0.378$ for females for the sex parameter; $\mathrm{r}=0.375$ for the 1st grade for the class parameter) and middle (sex parameter for males, $\mathrm{r}=0.418$; for the grade parameter, $\mathrm{r}=0.478$ for the second grade) were determined to be graded correlations $(p<0.01$; Table 4$)$.

The authors found the responses to the first question (Do you think you have a problem with the jaw/jaw joint?), from the three questions asked to individuals to determine their awareness of TMD or the treatments they received showed a statistically different distribution in individuals with and without TMD (Table 5; $p=0.030)$. When the question "Do you think you have a problem with the jaw/jaw joint?" was asked to individuals with TMD, it was reported that $75.0 \%$ of the individuals with TMD answered "no." For individuals who do not have TMD, five individuals answered "yes" to the question "Do you think you have a problem with the jaw/jaw joint?" while 32 individuals answered "no" (Table 5). The correlation analysis between TMD and OBC scores according to the answers given to these three questions is presented in Table 6. It was determined that there was a moderately significant positive correlation between TMD and OBC scores in participants who answered the question "Do you think you have a problem with the jaw/jaw joint?" as both "yes" ( $\mathrm{r}=0.472)$ and "no" $(\mathrm{r}=0.404)$. However, for the questions "Have you applied to the dentist for jaw/jaw joint pain in the last 1 year?" ( $\mathrm{r}=0.428)$ and "Have you seen any exercise (mouthpiece, medication, etc.) due to jaw/jaw joint pain in the last year?" ( $\mathrm{r}=0.426)$, there was a moderate positive correlation between the participants who answered "no" to the other questions (Table 6).

The correlation between the total OBC and FAI scores was calculated for each of the 21 questions in the OBC (Table 7). Items 4 (Spearman's rho $=0.451, p<0.001)$ and 6 (Spearman's rho $=0.444, p<0.001)$ had a moderate and significant correlation with TMD severity. When the relationship between FAI and OBC scores was examined by linear regression analysis (Table 8), the established regression model was found to be statistically significant $(\mathrm{F}=79.599 ; p<0.001)$. It was determined that as the FAI score increased, the OBC score increased, and when the FAI score increased by one unit, the OBC score increased by 0.414 units 
$(p<0.001)$.

\section{Discussion}

The dental assistant education program's importance, which contributes to the easier and faster provision of dentistry applications, is gradually increasing. ${ }^{30}$ In this study conducted with students of the dental assistant program, the authors examined the prevalence of TMD and oral habits while evaluating the relationship between TMD and OBC. In this cross-sectional study, it was determined that the prevalence of TMD in dental assistant program students was $64.8 \%$, and one of the most important findings was that they had at least one oral parafunction, and the majority of them correlated with FAI scores.

This study was conducted with 105 students in total. As in many previous studies conducted in a similar population, most of the participants were female individuals. ${ }^{5,11,12,16,31}$ Subclinical symptoms of TMD have been investigated with FAI, which is used in the early diagnosis and detection of TMD to prevent the progression and early treatment of TMD symptoms, enabling the rapid and accessible collection of large amounts of data at the minimum cost used in many previous studies. ${ }^{5,19,32,33}$ Similar to previous studies conducted in various university student populations and reported TMD prevalence between 37-80\% 5,13-17,19,25, this study, which included only dental assistant program students, also found a high prevalence of TMD (64.8\%) compared to the prevalence of TMD absence $(35.2 \%)$. These results may be due to differences in populations such as genetic structure, physiological differences, sex distribution, or social demographic characteristics, and the anxiety caused by the COVID-19 pandemic at the time of the study. ${ }^{3,19,31,34}$ However, when an evaluation was made according to the severity of TMD, it was determined that most students experienced mild problems. Among the three questions asked to learn whether there is a problem in the jaw joint, the question "Do you think you have a problem with the jaw/jaw joint?" only differed according to the severity of TMD, and the majority of the participants, most individuals with mild TMD, answered no. The reason for this was thought to be that there was online education, and students were at their homes due to the COVID-19 pandemic in the relevant period.35,36 In addition, in the study by Isiekwe et al. ${ }^{37}$, individuals participating in the dental assistant training stated that their knowledge about COVID-19 was at a reasonable level, which may have enabled health sciences students to accept the anxiety related to the pandemic more quickly.

In the literature, it has been reported that parafunctional habits, day and night clenching, and grinding are associated with painful TMJ problems. ${ }^{38}$ It has been reported that the main cause of day and night clenching and parafunctional habits is mental stress. ${ }^{39}$ This current study found that increased parafunctional and oral habits were associated with TMJ problems, using the OBC questionnaire, a self-administered tool designed to evaluate oral activities that occur during wakefulness or sleep. ${ }^{40}$ In this study, when the OBC scores were compared, it was found that the values of female students were higher than those of male students. This shows that there is a positive correlation between the FAI and OBC scores. In the literature, it has been reported that oral habits, such as chewing at a low level and continuously, teeth grinding, and squeezing, increase muscle activity, causing fatigue and pain. ${ }^{41}$ It was determined that there was no significant difference between $\mathrm{OBC}$ scores according to the education period. It is considered that the reason for this was that the type of education in the dental assistant program is shorter (2 years) and less intense than the standard 5 -year dentistry education. ${ }^{42}$ It was determined that there was a moderately significant correlation between OBC score severity and FAI index in both sexes and both education periods, similar to the findings obtained in previous studies. ${ }^{5,16,19}$ In addition, the correlation between OBC scores and FAI index for the answers to the three questions asked in TMD awareness was observed to be moderate and significant in the "no" answer for all questions. The authors predicted that this was because most participants had mild TMD and thought they did not have joint problems. In addition, with the regression analysis applied during this study, the mathematical relationship between the OBC and FAI scores was defined.

For each OBC question, present study results regarding the correlation between the score value of that question and the FAI scores are similar to the findings presented by Karabicak and Kanik. ${ }^{19}$ A moderately significant correlation was found between OBC scores and FAI scores in individuals who grit their teeth during the day. Supporting this finding, it has been stated in previous studies ${ }^{19,43}$ that clenching can be seen frequently during the day. It is also known that this clenching can be affected by the tension that varies 
during the day in individuals. ${ }^{44}$

Early diagnosis of TMD and the factors that cause it (occlusal factors developed as a result of oral habits) are vital for dentistry. Early diagnosis and treatment of problems will prevent them from further progressing and becoming complicated and increase individuals' quality of life. These survey studies, which seem simple, will increase the chance of treatment by increasing the prevalence, awareness, and early diagnosis of TMD in this respect.

\section{Limitations}

Only specific subclinical symptoms related to TMJ problems could be obtained when only one questionnaire was screened. In light of the data obtained, it is necessary to precisely diagnose TMD by clinical and radiological examinations and be treated afterward. This study was conducted only with students in a dental assistant program. When the students included in this study were evaluated in terms of sex distribution, there was a difference between the sexes in terms of numbers. However, it has been reported that the prevalence of TMD is higher in females. This situation may have affected the results of the study and made it difficult to generalize the results. Therefore, in future studies, it is necessary to evaluate TMJ problems and clinical and radiological examinations with similar but more participants and use different student populations, especially dental students.

\section{Conclusion}

In this study, a high prevalence of TMD (64.8\%) was found among dental assistant program students. It was observed that the rate of TMD was higher in female than in male students. According to the OBC score, each participant had at least one oral parafunction. Oral habits were moderately associated with TMD prevalence. Accordingly, students who are observed to have severe TMD scores should be guided by clinical and radiological examinations to begin appliance or clinical treatment procedures.

\section{ACKNOWLEDGEMENTS}

\section{CONFLICT OF INTEREST}

The authors declare that there are no conflicts of interest regarding the publication of this article.

\section{FUNDING INFORMATION}

The authors did not receive any funding support for the study.

\section{AUTHORS' CONTRIBUTIONS}

All authors contributed to the data analysis and interpretation as well as writing the manuscript and revising it critically and have given final approval of the version to be published. TO planned and performed the data collection.

\section{DATA AVAILABILITY STATEMENT}

Data available on request due to privacy/ethical restrictions.

\section{References}

1. Augusto VG, Perina KCB, Penha DSG, et al. Temporomandibular Dysfunction, Stress and Common Mental Disorder in University Students. Acta Ortop Bras 2016;24:330-333.

2. Westersund CD, Scholten J, Turner RJ. Relationship between craniocervical orientation and center of force of occlusion in adults. Cranio 2017;35:283-289.

3. Pedroni C, De Oliveira A, Guaratini M. Prevalence study of signs and symptoms of temporomandibular disorders in university students. J Oral Rehabil 2003;30:283-289.

4. Mehta NM. The role of interprofessional education in the management of temporomandibular and sleep disorders. Cranio 2013;31:159-160. 
5. Karaman A, Sapan Z. Evaluation of temporomandibular disorders, quality of life, and oral habits among dentistry students. Cranio. 2020:1-7.

6. Murphy GJ. TMD? TMD? What ever happened to the tops of the teeth? Cranio 2006;24:76-77.

7. Poveda Roda R, Bagán JV, et al. Review of temporomandibular joint pathology: Part I: Classification, epidemiology and risk factors. Med Oral Patol Oral Cir Bucal 2007;12:292-298.

8. Nekora-Azak A, Evlioglu G, Ceyhan A, et al. Estrogen replacement therapy among post-menopausal women and its effects on signs and symptoms of temporomandibular disorders. Cranio 2008;26:211-215.

9. Banafa A, Suominen AL, Sipilä K. Factors associated with signs of temporomandibular pain: an 11year-follow-up study on Finnish adults. Acta Odontol Scand 2020;78:57-63.

10. Schmid-Schwap M, Bristela M, Kundi M, et al. Sex-specific differences in patients with temporomandibular disorders. J Orofac Pain 2013;27:42-50.

11. Lee J-Y, Kim Y-K, Kim S-G, et al. Evaluation of Korean teenagers with temporomandibular joint disorders. J Korean Assoc Oral Maxillofac Surg 2013;39:231-237.

12. Bevilaqua-Grossi D, Chaves TC, de Oliveira AS, et al. Anamnestic index severity and signs and symptoms of TMD. Cranio 2006;24:112-118.

13. Nomura K, Vitti M, Oliveira ASd, et al. Use of the Fonseca's questionnaire to assess the prevalence and severity of temporomandibular disorders in Brazilian dental undergraduates. Braz Dent J 2007;18:163167.

14. Kaynak BA, Serkan T, Ünlüer NÖ, et al. Investigation of Prevalence of Temporomandibular Joint Dysfunction in University Students. Hacettepe University Faculty of Health Sciences Journal 2019;6:287298.

15. Özdinç SP, Ata HM, Selçuk HM, et al. Temporomandibular joint disorder determined by Fonseca anamnestic index and associated factors in 18- to 27-year-old university students. Cranio 2020;38:327332.

16. Türken R, Büyük SK, Yaşa Y. Evaluation of Temporomandibular Joint Disorders And Oral Habits in Dentistry Faculty Students. ACU Sağllk Bil Derg 2020;11:208-213.

17. Dervis NE. Prevalence of temporomandibular disorder in Turkish university students: A questionnaire study. Balk J Dent Med 2019;23:80-87.

18. Marklund S, Wänman A. Incidence and prevalence of myofascial pain in the jaw-face region. A one-year prospective study on dental students. Acta Odontol Scand 2008;66:113-121.

19. Karabicak GO, Hazar Kanik Z. Temporomandibular disorder prevalence and its association with oral parafunctions, neck pain, and neck function in healthcare students: A cross-sectional study. Cranio 2020:1-7.

20. LeResche L, Mancl LA, Drangsholt MT, et al. Predictors of onset of facial pain and temporomandibular disorders in early adolescence. Pain 2007;129:269-278.

21. Paduano S Md DDS, Bucci R Dds P, Rongo R Dds P, et al. Prevalence of temporomandibular disorders and oral parafunctions in adolescents from public schools in Southern Italy. Cranio 2020;38:370-375.

22. Manfredini D, Lobbezoo F. Relationship between bruxism and temporomandibular disorders: a systematic review of literature from 1998 to 2008. Oral Surg Oral Med Oral Pathol Oral Radiol Endod 2010;109:e26-e50.

23. Goldstein BH. Temporomandibular disorders: a review of current understanding. Oral Surg Oral Med Oral Pathol Oral Radiol Endod 1999;88:379-385.

24. Oliveira ASd, Dias EM, Contato RG, et al. Prevalence study of signs and symptoms of temporomandibular disorder in Brazilian college students. Braz Oral Res 2006;20:3-7.

25. Kaynak BA, Taş S, Salkın Y. The accuracy and reliability of the Turkish version of the Fonseca anamnestic index in temporomandibular disorders. Cranio 2020:1-6.

26. Lobbezoo F, Lavigne GJ. Do bruxism and temporomandibular disorders have a cause-and-effect relationship? Journal of orofacial pain 1997;11:15-23.

27. Bonta G, Campus G, Cagetti MG. COVID-19 pandemic and dental hygienists in Italy: a questionnaire survey. BMC Health Serv Res 2020;20:994.

28. Antoun JS, Mei L, Gibbs K, Farella M. Effect of orthodontic treatment on the periodontal tissues. 
Periodontol 2000 2017;74:140-157.

29. Schober P, Boer C, Schwarte LA. Correlation coefficients: appropriate use and interpretation. Anesth Analg 2018;126:1763-1768.

30. Kracher C, Breen C, McMahon K, et al. The evolution of the dental assisting profession. J Dent Educ 2017;81:eS30-eS37.

31. Medeiros RAD, Vieira DL, Silva EVFD, et al. Prevalence of symptoms of temporomandibular disorders, oral behaviors, anxiety, and depression in Dentistry students during the period of social isolation due to COVID-19. J Appl Oral Sci 2020;28:e20200445.

32. Topuz MF, Oghan F, Ceyhan A, et al. Assessment of the severity of temporomandibular disorders in females: Validity and reliability of the Fonseca anamnestic index. Cranio 2020:1-4.

33. Stasiak G, Maracci LM, de Oliveira Chami V, et al. TMD diagnosis: Sensitivity and specificity of the Fonseca Anamnestic Index. Cranio 2020:1-5.

34. Jerjes W, Madland G, Feinmann C, et al. A psychological comparison of temporomandibular disorder and chronic daily headache: are there targets for therapeutic interventions? Oral Surg Oral Med Oral Pathol Oral Radiol Endod 2007;103(3):367-373.

35. Deery C. The COVID-19 pandemic: implications for dental education. Evid Based Dent 2020;21:46-47.

36. Chang T-Y, Hong G, Paganelli C, et al. Innovation of dental education during COVID-19 pandemic. J Dent Sci 2021;16:15-20.

37. Isiekwe IG, Umeizudike KA, Daramola OO, et al. The COVID-19 pandemic and dental residency training in Nigeria. Eur J Dent Educ. 2020.

38. Fernandes G, van Selms MKA, Gonçalves DAG, et al. Factors associated with temporomandibular disorders pain in adolescents. J Oral Rehabil 2015;42:113-119.

39. Carra MC, Huynh N, Morton P, et al. Prevalence and risk factors of sleep bruxism and wake-time tooth clenching in a 7- to 17-yr-old population. Eur J Oral Sci 2011;119:386-394.

40. Kaplan SE, Ohrbach R. Self-Report of Waking-State Oral Parafunctional Behaviors in the Natural Environment. J Oral Facial Pain Headache 2016;30:107-119.

41. Koutris M, Lobbezoo F, Naeije M, et al. Effects of intense chewing exercises on the masticatory sensorymotor system. J Dent Res 2009;88:658-662.

42. Robinson DS, Bird DL. Introduction to Dental Assisting. In: Robinson DS, Bird DL (eds). Essentials of Dental Assisting-E-Book. 7th ed. St. Louis, Missouri: Elsevier Health Sciences, 2017:1-10.

43. Yalçın Yeler D, Yılmaz N, Koraltan M, et al. A survey on the potential relationships between TMD, possible sleep bruxism, unilateral chewing, and occlusal factors in Turkish university students. Cranio 2017;35:308-314.

44. Karthik R, Hafila MF, Saravanan C, et al. Assessing prevalence of temporomandibular disorders among university students: a questionnaire study. J Int Soc Prev Community Dent 2017;7:S24-S29.

Figure Legend

Figure 1. Flow diagram of the participants included in the study. 


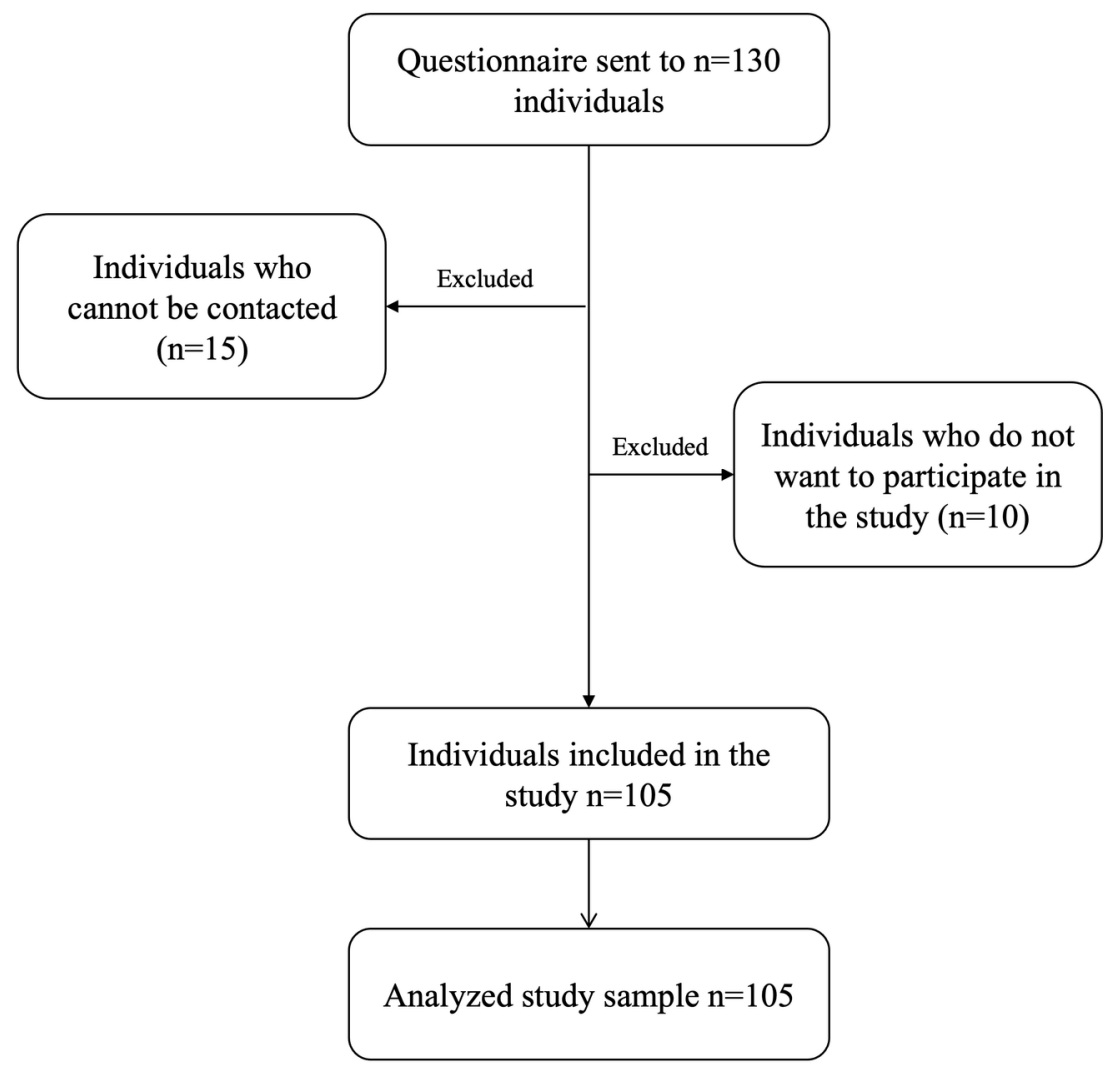

\section{Hosted file}

Table 1.docx available at https://authorea.com/users/423854/articles/529082-evaluationof-temporomandibular-disorder-and-oral-parafunction-prevalence-in-dental-assistantstudents-a-cross-sectional-descriptive-study

\section{Hosted file}

Table 2.docx available at https://authorea.com/users/423854/articles/529082-evaluationof-temporomandibular-disorder-and-oral-parafunction-prevalence-in-dental-assistantstudents-a-cross-sectional-descriptive-study

\section{Hosted file}

Table 3.docx available at https://authorea.com/users/423854/articles/529082-evaluationof-temporomandibular-disorder-and-oral-parafunction-prevalence-in-dental-assistantstudents-a-cross-sectional-descriptive-study

\section{Hosted file}

Table 4.docx available at https://authorea.com/users/423854/articles/529082-evaluationof-temporomandibular-disorder-and-oral-parafunction-prevalence-in-dental-assistantstudents-a-cross-sectional-descriptive-study

\section{Hosted file}

Table 5.docx available at https://authorea.com/users/423854/articles/529082-evaluationof-temporomandibular-disorder-and-oral-parafunction-prevalence-in-dental-assistantstudents-a-cross-sectional-descriptive-study 


\section{Hosted file}

Table 6.docx available at https://authorea.com/users/423854/articles/529082-evaluationof-temporomandibular-disorder-and-oral-parafunction-prevalence-in-dental-assistantstudents-a-cross-sectional-descriptive-study

\section{Hosted file}

Table 7.docx available at https://authorea.com/users/423854/articles/529082-evaluationof-temporomandibular-disorder-and-oral-parafunction-prevalence-in-dental-assistantstudents-a-cross-sectional-descriptive-study

\section{Hosted file}

Table 8.docx available at https://authorea.com/users/423854/articles/529082-evaluationof-temporomandibular-disorder-and-oral-parafunction-prevalence-in-dental-assistantstudents-a-cross-sectional-descriptive-study 